Received: 31 October 2018

Accepted: 30 May 2019

Published online: 25 June 2019
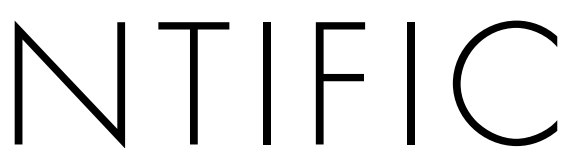

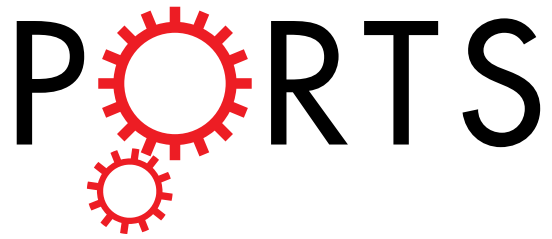

\title{
OPEN
}

\section{Transcriptomic analysis of 3D Cardiac Differentiation of Human Induced Pluripotent Stem Cells Reveals Faster Cardiomyocyte} Maturation Compared to 2D Culture

\author{
Mariana A. Branco ${ }^{1,2}$, João P. Cotovio ${ }^{1,2}$, Carlos A. V. Rodrigues ${ }^{1,2}$, Sandra H. Vaz ${ }^{3,4}$, \\ Tiago G. Fernandes ${ }^{1,2}$, Leonilde M. Moreira ${ }^{1}$, Joaquim M. S. Cabral ${ }^{1,2}$ \& \\ Maria Margarida Diogo ${ }^{1,2}$
}

Human induced pluripotent stem cells (hiPSCs) represent an almost limitless source of cells for disease modelling and drug screening applications. Here we established an efficient and robust 3D platform for cardiomyocyte (CMs) production from hiPSCs, solely through small-molecule-based temporal modulation of the Wnt signalling, which generates more than $90 \% \mathrm{cTNT}^{+}$cells. The impact of performing the differentiation process in 3D conditions as compared to a 2D culture system, was characterized by transcriptomic analysis by using data collected from sequential stages of $2 \mathrm{D}$ and 3D culture. We highlight that performing an initial period of hiPSC aggregation before cardiac differentiation primed hiPSCs towards an earlier mesendoderm lineage differentiation, via TGF- $\beta /$ Nodal signaling stabilization. Importantly, it was also found that CMs in the 3D microenvironment mature earlier and show an improved communication system, which we suggested to be responsible for a higher structural and functional maturation of 3D cardiac aggregates.

Human embryonic stem cells (hESCs) and human induced pluripotent stem cells (hiPSCs), collectively referred as human pluripotent stem cells (hPSCs), offer an almost limitless source of cells for clinical translation applications. Particularly, cardiomyocytes (CMs) obtained from in vitro differentiation of hiPSCs have been considered an attractive tool for disease modelling and drug screening applications ${ }^{1,2}$.

The identification of key signalling pathways and the transcriptional network linked to embryonic heart development, guided the establishment of a number of in vitro models for cardiac differentiation from hPSCs. Through the sequential addition of growth factors and/or small molecules, the critical stages of cardiac specification have been recapitulated to some extent using $2 \mathrm{D}$ in vitro models ${ }^{3-6}$. However, human heart development is a complex process in which spatial gradients of molecules and biophysical stimuli, due to the three-dimensional (3D) configuration of the embryo, are crucial to determine the final heart tissue structure and function ${ }^{7,8}$. Therefore, these processes are not well recapitulated in the commonly used monolayer (2D) differentiation platforms.

Aiming at better mimicking the microenvironment of in vivo heart development, 3D platforms for in vitro cardiac differentiation and maturation have emerged in the past few years. However, and despite the existence of different reported protocols for hPSC differentiation into CMs as 3D aggregates ${ }^{9-12}$, the development of an efficient, controlled and reproducible process of 3D differentiation has been challenging.

${ }^{1}$ Department of Bioengineering and iBB-Institute for Bioengineering and Biosciences, Instituto Superior Técnico, Universidade de Lisboa, 1049-001, Lisbon, Portugal. ${ }^{2}$ The Discoveries Centre for Regenerative and Precision Medicine, Lisbon Campus, Instituto Superior Técnico, Universidade de Lisboa, 1049-001, Lisbon, Portugal. ${ }^{3}$ Instituto de Medicina Molecular, Faculdade de Medicina, Universidade de Lisboa, 1649-028, Lisbon, Portugal. ${ }^{4}$ Instituto de Farmacologia e Neurociências, Faculdade de Medicina da Universidade de Lisboa, 1649-028, Lisbon, Portugal. Correspondence and requests for materials should be addressed to M.M.D. (email: margarida.diogo@tecnico. ulisboa.pt) 
A

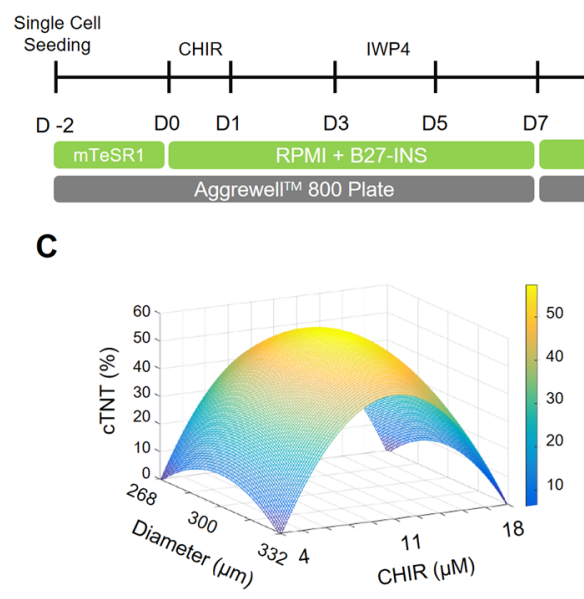

B
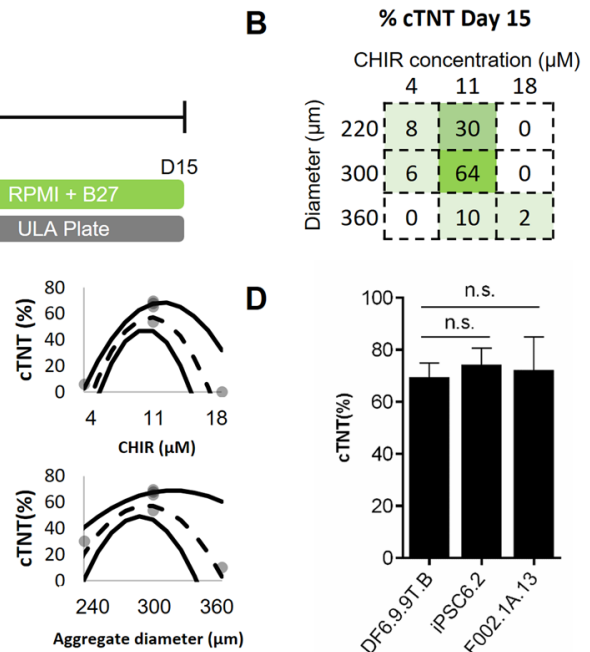

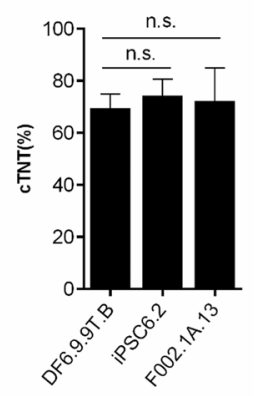

Figure 1. Forced aggregation of hiPSCs in microwells allows efficient generation of 3D cardiac tissue. (A) Schematic representation of cardiomyocyte differentiation from hiPSCs in a 3D culture system, using the temporal modulation of Wnt signalling. AggreWell ${ }^{\mathrm{TM}} 800$ plates were used to obtain size-controlled aggregates. ULA - Ultra Low Attachment plates. (B) Percentage of cTNT ${ }^{+}$cells after 15 days of differentiation for the experimental runs of the factorial design. CHIR concentration ranges from $4 \mu \mathrm{M}$ to $18 \mu \mathrm{M}$ (centre in $11 \mu \mathrm{M}$ ), and aggregate diameter between $220 \mu \mathrm{m}$ and $360 \mu \mathrm{m}$ (centred in $300 \mu \mathrm{m}$ ). Each of the tested combinations was performed once, excluding the centred point of the factorial design in which $n=4$ independent experiments were performed. (C) $3 \mathrm{D}$ representation of the quadratic model relating initial aggregate diameter and small molecule CHIR concentration with the percentage of $\mathrm{cTNT}^{+} \mathrm{CMs}$ after 15 days of differentiation.

(D) Validation of the optimal conditions (aggregate size: $290 \mu \mathrm{M}$; CHIR concentration: $11 \mu \mathrm{M}$ ) with DF6.9.9 T.B cell line and two other hiPSC lines (F002.1 A.13 and iPSC6.2). Data are represented as mean \pm SEM, $n=3$ independent experiments for F002.1 A.13 and iPSC6.2 lines and $n=6$ independent experiments for DF6.9.9 T.B cell line. n.s. - No statistically significant (p-value $>0.05$ ). See also Fig. S1.

3D culture of hiPSCs has been shown to favor transcriptional changes that improve differentiation into specific lineages ${ }^{13-16}$, but the mechanisms behind this effect have not yet been completely understood. Furthermore, the development of a platform that takes advantage of the reported knowledge regarding $3 \mathrm{D}$ culture of hiPSCs to establish a robust and straightforward cardiac differentiation protocol has not yet been reported. Additionally, the sole impact that 3D culture exerts throughout the process of hiPSC-CM differentiation, from the moment 3D aggregates are generated until the stage of CM maturation remains also poorly understood. As an example of the relevance of the culture format in this process, a recent study that performed $3 \mathrm{D}$ aggregation of cardiac progenitor cells obtained in 2D culture system, showed the benefits of 3D culture at earlier stages of cardiac differentiation regarding structural and metabolic maturation of the final $\mathrm{CMs}^{17}$.

In this work, we expanded the knowledge regarding the impact of 3D culture of hiPSCs in a forced aggregation platform and took advantage of that knowledge to develop a simple, efficient and robust 3D platform for hiPSC differentiation towards CMs, using the temporal modulation of the Wnt signalling pathway. RNA sequencing (RNA-seq) was used to generate global gene expression profiles for sequential stages of cardiac differentiation of both 3D aggregates and parallel monolayer 2D culture conditions. Expression profiling data analyses revealed that the initial period of hiPSC 3D aggregation before cardiac differentiation induces significant transcriptional changes that favour the cardiac differentiation process by priming hiPSCs to mesendoderm lineages. Also, the obtained data suggests that the CMs obtained in this 3D microenvironment mature earlier when compared with 2D cardiac monolayer.

\section{Results}

Forced aggregation of hiPSCs on microwells allows efficient generation of cardiomyocytes. To develop a platform for 3D cardiac differentiation of hiPSCs, we used the temporal modulation of Wnt signalling pathway ${ }^{18}$ and a factorial design approach ${ }^{19}$ for the optimization process. In order to generate size-controlled aggregates, forced aggregation of single hiPSCs in the commercially available AggreWell ${ }^{\mathrm{TM}} 800$ plates was performed. Aggregate size was controlled using different cell seeding densities (Fig. S1A), and hiPSC aggregates were maintained during 48 hours in $\mathrm{mTeSR}^{\mathrm{TM}} 1$, before starting the differentiation process (D0) (Fig. 1A).

The cardiac differentiation process was optimized by testing the combined effect of small molecule CHIR concentration, ranging from $4 \mu \mathrm{M}$ to $18 \mu \mathrm{M}$, and aggregate diameter at day 0 , ranging from $220 \mu \mathrm{m}$ to $360 \mu \mathrm{m}$, with the model centred in the culture condition corresponding to $11 \mu \mathrm{M}$ CHIR and $300 \mu \mathrm{m}$, respectively. Cardiomyocyte differentiation efficiency was evaluated as the percentage of cells expressing the cardiomyocyte marker cardiac troponin T (cTNT) at day 15 of differentiation. The experimental results obtained for the different tested conditions are represented in Fig. 1B. The centred condition $(11 \mu \mathrm{M} \mathrm{CHIR,} 300 \mu \mathrm{m})$ was the one that yielded the highest percentage of $\mathrm{cTNT}^{+}$cells after 15 days of differentiation, resulting in an average of $64 \pm 4 \%$ 
$\mathrm{cTNT}^{+}$cells (Fig. 1B and Fig. S1B). From the quadratic model generated from the experimental results (Fig. 1C and Fig. S1C), only the quadratic term of CHIR concentration and aggregate diameter had statistical significance (Fig. S1D), which resulted in a maximum value for the considered output of the model (cTNT ${ }^{+}$cells at day 15) for a specific aggregate diameter and CHIR concentration. The optimal condition that allowed reaching a percentage of $\mathrm{cTNT}^{+}$cells between $50-66 \%$ was obtained when initiating the integrated process with an aggregate diameter of $289 \pm 12 \mu \mathrm{m}$ and a CHIR concentration of $10.8 \pm 0.5 \mu \mathrm{M}$, which is in fact close to the centred point used in the factorial design.

The optimized cardiac differentiation platform was validated using the DF6-9-9T.B cell line, the one used for the factorial design optimization process, and two additional hiPSC lines, iPSC6.2 and F002.1 A.13, proving the reproducibility and robustness of the platform (Fig. 1D). Additionally, a time-course analysis of cardiac differentiation markers until day 20 was performed, confirming the normal progression of cardiac differentiation (Fig. S1E). In addition to the parameters that were optimized through the factorial design, it was also verified that by decreasing to 24 hours (D-1) the culture period before initiating the differentiation, the efficiency in terms of percentage of cardiomyocytes drastically dropped (Fig. S1F), reinforcing the importance of the 3D hiPSC aggregates expansion period.

\section{D culture of hiPSCs under pluripotency maintenance medium primes hiPSCs towards mes-} endoderm lineage. In order to reveal the main impact of $3 \mathrm{D}$ culture towards cardiac differentiation, a transcriptomic analysis was performed (Table S1), using 2D cardiac differentiation, based also on the temporal modulation of the Wnt signalling, as control ${ }^{5,20}$.

The first stage of hiPSC culture before cardiac induction involves a short period of hiPSCs expansion for both $2 \mathrm{D}$ and $3 \mathrm{D}$ culture conditions (Fig. 2A), which from now on will be designated as the pre-differentiation period. As confirmed by principal component analysis (PCA) (Fig. 2B), gene expression profile of D0 hiPSC population for both 2D ("2D-D0") and 3D ("3D-D0") differentiation culture formats, showed considerable differences when compared with the "hiPSC seeding" population (initial hiPSCs seeded in 2D and 3D platforms), mainly discriminated by PC2 (16\% of total variance), which is probably related with the degree of cell confluence and/or cell communication. Differences at gene expression level between "3D-D0" and "2D-D0" are also evident, and were mainly discriminated by PC1 (58\% of total variance), which explains the majority of the observed differences in the analysed dataset and seems to be linked with the culture format (Fig. 2B and Fig. S2A).

To understand those differences, a comparative analysis between "3D-D0" and "hiPSC seeding" expression profiling was performed (Table S2 "DE gene list"). Gene ontology (GO) analysis using the upregulated genes shows that the most enriched biological process in the 3D culture format was related with response to hypoxia (Fig. 2C and Table S2 "GO - 3D D0 vs hiPSC seeding - up"). Since 3D hiPSC aggregates had already $\approx 290 \mu \mathrm{m}$ of diameter at D0, it is not surprising the activation of genes involved in hypoxic response as a result of oxygen diffusion limitations throughout the entire aggregate ${ }^{21}$. To demonstrate the existence of an hypoxic environment inside the 3D aggregates, the expression level of the HIF1 $\alpha$ protein, which is the master transcriptional regulator of hypoxic response 22 $^{2}$ was quantified in both "2D-D0" and "3D-D0" cell populations. The results revealed a significant increase in HIF1 $\alpha$ protein expression levels in 3D aggregates as compared to the 2D monolayer (Fig. S2B). Since gradients of oxygen and nutrients inside the 3D aggregates could potentially compromise cell viability, we quantified the percentage of viable cells in 3D-D0 aggregates and compared with "2D-D0" population, confirming that the viability of the cells was not affected by $3 \mathrm{D}$ culture (Fig. S2C).

To highlight the genes that were upregulated in the "3D-D0" cell population vs "hiPSC seeding" population, a volcano plot was generated (Fig. 2D). Among the upregulated genes, PGK1, LDHA, BNIP3 and JUN stand out, within the group of genes that show a higher and more significant upregulation. The higher expression level of those genes in 3D-D0 aggregates, when compared not only to "hiPSC seeding" cells but also to 2D-D0 monolayer cells, was confirmed by qRT-PCR, (Fig. 2E). In agreement with the hypothesis of an hypoxic response inside the $3 \mathrm{D}$ aggregates, the aforementioned genes are known direct or indirect targets of HIF $1 \alpha$ activation network. Specifically, PGK1 and $L D H A$ are genes involved in glycolysis, which suggests that in the $3 \mathrm{D}$ environment the rate of glycolysis is higher compared with both "2D-D0" and "hiPSC seeding" conditions. GO analysis of the downregulated genes in "3D-D0" and "2D-D0" environments compared to "hiPSC seeding" population, revealed that some of the downregulated genes in 3D-D0 population are related with the oxidative phosphorylation and mitochondrial respiratory chain (Fig. S2D,E), reinforcing the proposal of a higher rate of glycolysis in 3 D-D0 aggregates. Finally, glucose consumption and lactate production rates were analysed in both "2D-D0" and "3D-D0" conditions. The results revealed an equivalent specific glucose consumption rate in 2D-D0 and 3D-D0 conditions, however a statistically significant higher specific lactate production rate in hiPSCs present in the 3D-D0 aggregates was observed when compared with the cells in 2D monolayer. This results on a higher yield of lactate production/glucose consumption, which is indicative of a higher level of glycolysis in 3D-D0 aggregates (Fig. S2F). In fact, hypoxia is a known trigger of metabolic changes in hPSCs and it is also described to be involved in pluripotency maintenance by promoting glycolysis and preventing mitochondrial respiration ${ }^{23,24}$.

The activation of MAPK/JNK/ERK signalling is also evident in "3D-D0" aggregates when compared with "hiPSC seeding" and "2D-D0", with the upregulation of JUN, FOSB, FOS and EGR1 genes. The possible activation of this pathway, which is involved in the transcription of a wide range of cell proliferation and apoptotic genes ${ }^{25}$, can be also a consequence of reduced oxygen levels, specifically due to increased concentration of ROS inside the cells ${ }^{26}$. Additionally, JNK/ERK signalling pathway can be indirectly activated as a result of the increased cytosolic concentration of $\mathrm{Ca}^{2+}$ triggered by $\mathrm{ROS}^{27}$, since $\mathrm{Ca}^{2+}$ has been described to be involved in MAPK/JNK/ ERK signalling activation ${ }^{28}$. In fact, the calcium voltage channel Cav1.2 encoded by $C A C N A 1 C$, is upregulated in 3D-D0 aggregates, as well as CXCR4, which is described to be involved in the regulation of $\mathrm{Ca}^{2+}$ mobilization and activation of MAPK signalling ${ }^{29}$. MAPK/JNK pathway had been reported to be involved in the maintenance of 
A

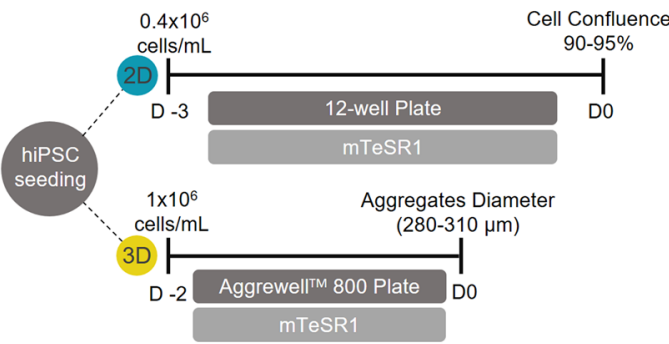

B

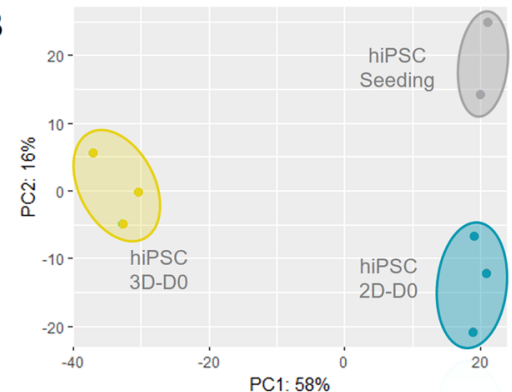

C

3D-D0 vs hiPSC Seeding

D 3D-D0 vs hiPSC Seeding

E
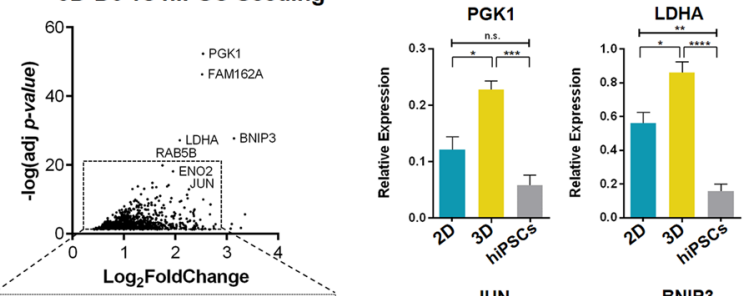

response to oxidative stress (GO:0006979)

response to reactive oxygen species (GO:0000302)

cellular response to hypoxia (GO:0071456)

system development (GO:0048731)

response to mechanical stimulus (GO:0009612)

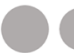

542

$-\log 2$ (FDR)

$\mathbf{F}$

3D-D0 vs 2D-D0

primitive streak formation (GO:0090009)

developmental process (GO:0032502)

anterior/posterior axis specification (GO-0009948)

SMAD protein signal transduction (GO:0060395)

cell differentiation (GO:0030154)

$$
\begin{array}{lll}
10 & 6 & \\
-\log 2(F D R)
\end{array}
$$
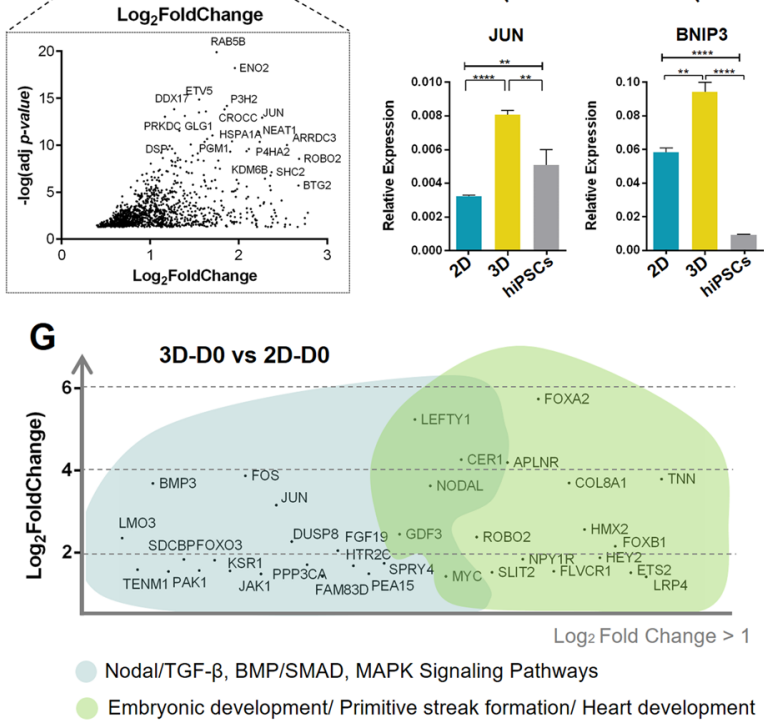

H
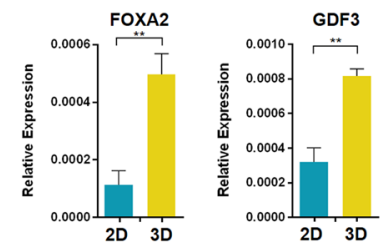
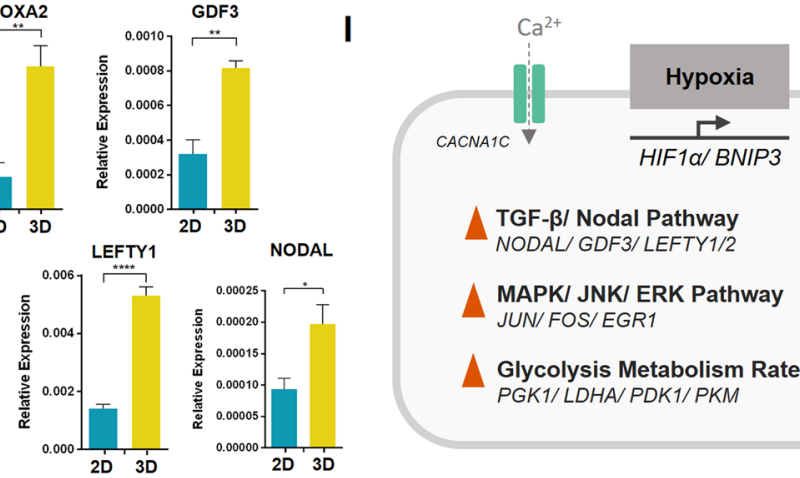

MAPK/ JNK/ ERK Pathway JUN/FOS/EGR1

Glycolysis Metabolism Rate PGK1/ LDHA/ PDK1/PKM

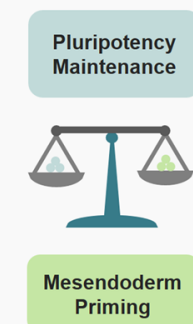

Figure 2. 3D culture of hiPSCs under pluripotency maintenance medium primes hiPSCs towards mesendoderm lineage. (A) Schematic representation of hiPSC culture, before cardiac differentiation, in both $2 \mathrm{D}$ and $3 \mathrm{D}$ culture strategies. In $2 \mathrm{D}$ monolayer, hiPSCs were seeded at a density of $0.4 \times 10^{6} \mathrm{cells} / \mathrm{mL}$, reaching 90-95\% confluence after 3 days of culture. In the 3D culture platform, hiPSCs were seeded at a density of $1 \times 10^{6} \mathrm{cells} / \mathrm{mL}$, reaching at day 0 of differentiation a diameter ranging from 280 to $310 \mu \mathrm{m}$. (B) PCA based on bulk RNA-seq data from three different conditions: hiPSCs used for seeding (hiPSC seeding, common for both culture strategies) $(\mathrm{n}=2$, independent experiments), 3D day 0 (3D-D0) and 2D day $0(2 \mathrm{D}-\mathrm{D} 0)(\mathrm{n}=3$, independent experiments). (C) Top gene ontology (GO) biological processes terms identified (FDR $<0.05)$ for the differentially upregulated genes $\left(\log _{2} \mathrm{FC}>2\right.$ and adjusted $p$-value $\left.<0.05\right)$ of 3D-D0 versus hiPSC seeding. (D) Volcano Plot highlighting the most significant upregulated genes for 3D-D0 versus hiPSC seeding populations ( $\log _{2}$ FoldChange $>1$ and Adjusted $p$-value $\left.>0.05\right)$. (E) Expression profile of PGK1, LDHA, BNIP3 and JUN genes for the three different populations: 3D-D0, 2D-D0 and hiPSC seeding. Values are normalized 
to $G A P D H$. Data are represented as mean \pm SEM, of at least $\mathrm{n}=3$ independent experiments. (F) Top GO biological processes terms identified $(\mathrm{FDR}<0.05)$ for the differentially upregulated genes $\left(\log _{2} \mathrm{FC}>2\right.$ and adjusted p-value $<0.05$ ) of 3D-D0 versus 2D-D0 comparison. See Table S2 for full DE gene list. (G) Graphical representation highlighting a set of the upregulated genes in 3D-D0 versus 2D-D0 comparison, related with the enriched biological processes "Embryonic development/Primitive streak formation/Heart development" and "Nodal/TGF- $\beta$, BMP/SMAD and MAPK Signaling Pathways", which stands out from the GO analysis $\left(\log _{2}\right.$ FoldChange $>1$ and adjusted p-value $>0.05$ ). (H) Expression profile of FOXA2, GDF3, CER1, NODAL and LEFTY1 genes in 3D-D0 and 2D-D0 populations. Values are normalized to GAPDH. Data are represented as mean \pm SEM, of at least $\mathrm{n}=3$ independent experiments. (I) Schematic representation of the proposed signalling network involved in hiPSCs culture as 3D aggregates in a forced aggregation platform. Oxygen gradients inside the spheroids trigger a hypoxic stimuli which is responsible for cell adaptation through (1) stabilization of TGF- $\beta$ /Nodal pathway, (2) upregulation of MAPK/JNK/ERK pathway and (3) increase in glycolysis metabolism, culminating in a balance between pluripotency maintenance and hiPSCs priming towards differentiation, particularly into mesendoderm lineage (Fig. 2I). See also Fig. S2.

stemness of hPSCs but activation of this pathway has also been reported to be linked with the initiation of hPSC differentiation ${ }^{30}$.

Additionally, a differential gene expression analysis between "3D-D0" and "2D-D0" was performed (Table S2 "DE gene list"). From the GO analysis focused on the most significantly upregulated genes, the main differences were related with regulation of Nodal signalling, SMAD protein signal transduction and the induction of primitive streak (PS) (Fig. 2F and Table S2 "GO - 3D-D0 vs 2D-D0 - up"). Within the upregulated genes, ligands of Nodal signalling, including NODAL and GDF3, as well as their direct targets, LEFTY1, LEFTY2 and CER1, that act in a negative feedback loop ${ }^{31}$, stand out (Fig. 2G). The upregulation of some of these genes, namely GDF3, CER1, LEFTY1 and NODAL, was confirmed by qRT-PCR, corroborating the RNA-seq data (Fig. 2H). Interestingly, Nodal signalling is not upregulated in "3D-D0" vs "hiPSC seeding" and instead it is down-regulated in "2D-D0" vs "hiPSC seeding", meaning that 3D culture of hiPSCs potentially induced the stabilization of Nodal signaling.

Although Activin/Nodal signalling is described to be involved in pluripotency maintenance of hPSCs, it is also described to be responsible for driving early cell fate decisions along the mesendodermal lineages ${ }^{31}$. The upregulation of FOXA2 in "3D-D0" aggregates compared with "2D-D0" (Fig. 2H), which is expressed in early/ anterior PS ${ }^{32}$, combined with TGF- $\beta /$ Nodal signalling activity, strengthens the hypothesis of hiPSC priming to mesendoderm lineages in $3 \mathrm{D}$ conditions.

In summary, our results suggest that hiPSCs culture as 3D aggregates in a forced aggregation platform results in oxygen gradients inside the spheroids, which are responsible for cell adaptation through (1) stabilization of TGF- $\beta$ /Nodal pathway, (2) upregulation of MAPK/JNK/ERK pathway and (3) increase in glycolysis metabolism, culminating in a balance between pluripotency maintenance and hiPSC priming towards differentiation, particularly into mesendoderm lineage (Fig. 2I).

3D cardiac differentiation allows a faster structural and functional maturation of hiPSC-CMs when compared with the 2D platform. To understand the impact of hiPSC 3D culture during the process of cardiac differentiation, we analysed the differentially expressed genes in 3D aggregates throughout the differentiation process, when compared with the $2 \mathrm{D}$ monolayer differentiation. Using a set of known genes involved in cardiogenesis and cardiomyocyte maturation, a PCA analysis (Table S3 "PCA 3D and 2D (D0 -D20)") was performed, which highlights differences regarding the progression of cardiac differentiation in both culture systems (Fig. 3A).

During the first 7 days of cardiac differentiation, 3D aggregates remain inside the microwell platform, which seems to be responsible for a prolonged oxidative stress and activation of hypoxic response. The transcriptional activation of metallothionein (MT) protein-coding genes during the first days of $3 \mathrm{D}$ cardiac differentiation, including $M T 1 G, M T 1 H, M T 1 E, M T 2 A, M T 1 F$ and $M T 1 X$, which prevent cells from apoptosis ${ }^{33}$, reinforce the hypothesis of an oxidative stress response.

At day 1 of differentiation, GO analysis focused on the most significantly upregulated genes in 3D-D1 aggregates and 2D-D1 monolayer when compared to "3D-D0" and "2D-D0" initial populations, respectively (Fig. 3B), revealed that the most upregulated genes in "3D-D1" were statistically more related with "tissue development process" and "cardiac tissue specification" biological processes, and in "2D-D1" more related with "gastrulation", "primitive streak formation" and "anterior/posterior pattern specification" biological processes. This corroborates the idea that $3 \mathrm{D}$ aggregates at day 0 of differentiation are more primed for mesendoderm differentiation and, consequently, cardiac differentiation progresses faster. Focusing our analysis only on transcription factors (TFs), RNA-seq data indicates that TFs known to be related with cardiac differentiation, such as MESP1, GATA4, $T B X 3, M S X 2$, were already upregulated at D1 of differentiation in 3D aggregates (Fig. S3A). Additionally, a higher expression of the gene $T$, which is one of the most important genes involved in mesendoderm specification, in 3D-D1 aggregates when compared with 2D-D1 monolayer, was further confirmed by qRT-PCR analysis (Fig. S3B). Also, the Wnt/ $\beta$-catenin target genes, AXIN2 and DKK1, were statistically significantly upregulated in the $3 \mathrm{D}$ aggregates compared with $2 \mathrm{D}$ monolayer at day 1 of differentiation, suggesting a higher degree of canonical Wnt signalling activation (Table S3 "DE gene list").

Moving along the cardiac differentiation process, at day 5 of differentiation a set of genes related with cardiac development and sarcomere structure and function were upregulated in the 3D aggregates (Table S3 "DE gene list”), including MEF2C, MYH6, ACTC1, ATP1A2, CACNA1D, RYR2, TNNT2 and TNNI1 (Fig. 3C). At day 
A

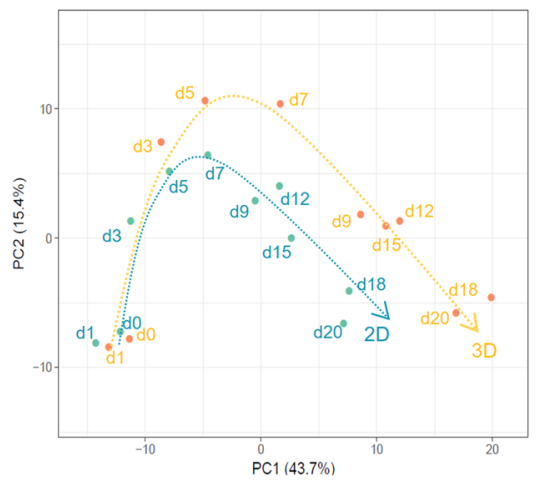

B

Gastrulation (GO:0007369)

Primitive streak formation (GO:0090009)

regionalization (GO:0003002)

pattern specification process (GO:0007389)

tissue development (GO:0009888)

developmental process (GO:0032502)

Embryonic morphogenesis (GO:0048598)

Heart development (GO:0007507)

Cardiac muscle tissue development (GO:0014706)

$\log _{2}$ Fold Change $>1$
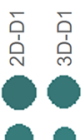

-

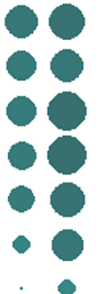

vs D0
C

\begin{tabular}{|c|c|c|}
\hline & Day 5 & $3 \mathrm{D}$ vs $2 \mathrm{D}$ \\
\hline \multirow{13}{*}{ 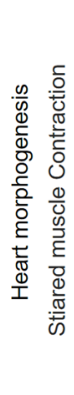 } & PLN & CACNA1D \\
\hline & MYH6 & TNNI1 \\
\hline & TNNT2 & SLC8A1 \\
\hline & TBX5 & RYR2 \\
\hline & XIRP1 & CREB5 \\
\hline & KCNA5 & MICAL2 \\
\hline & SMYD1 & CACNG6 \\
\hline & DHRS3 & PPP1R1A \\
\hline & MB & ATP1B2 \\
\hline & MEF $2 C$ & $\mathrm{KCNH} 2$ \\
\hline & $\mathrm{ACTC1}$ & PDGFA \\
\hline & TGFB2 & DSP \\
\hline & PROX1 & PPP2R5A \\
\hline
\end{tabular}

G $\quad \stackrel{\log _{2} \text { Fold Change }}{\stackrel{6}{\text { Enriched in } 3 \mathrm{D}}}$ Day 20

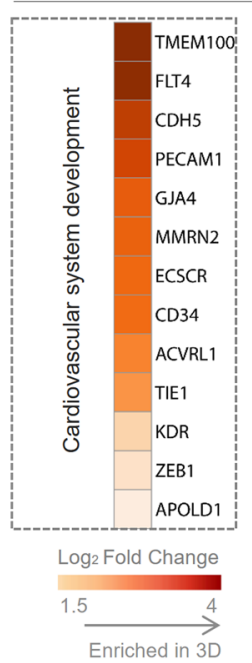

D

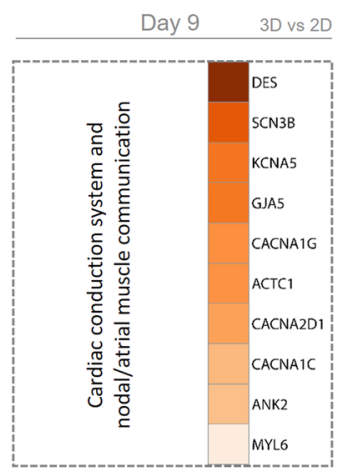

$\log _{2}$ Fold Change

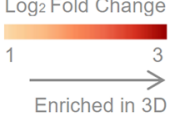

E

Beginning of contraction

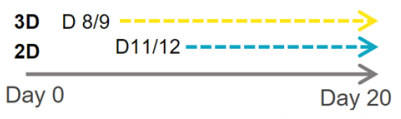

$\mathbf{F}$
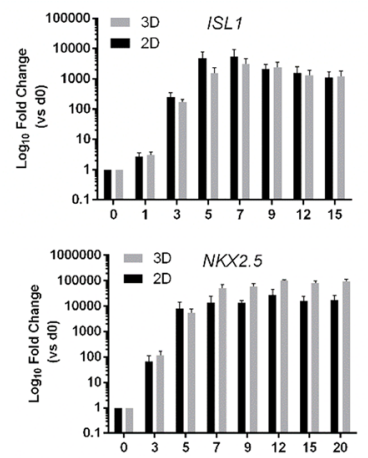

H

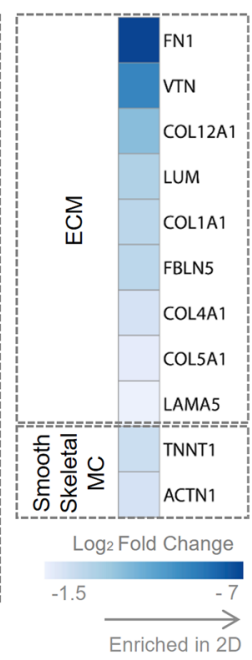

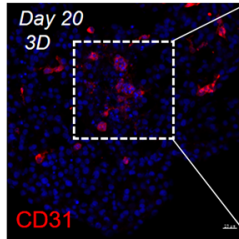

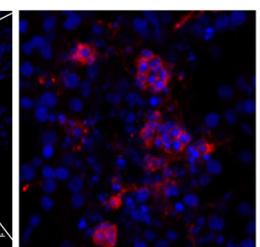

I
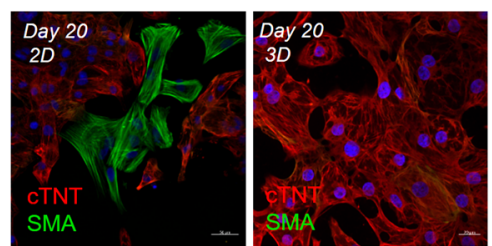

Figure 3. 3D cardiac differentiation allows a faster structural and functional maturation of hiPSC-CMs when compared with the 2D platform. (A) PCA of RNA-seq data in counts per million (CPM) using a subset of 254 genes linked to cardiac differentiation progression, CM functional and structural maturation, CM metabolism, Wnt, TGF- $\beta$ and FGF signalling pathways and other cardiac cells. See also Table S3 for more details. Orange and blue arrows describe the pathway followed by $3 \mathrm{D}$ and $2 \mathrm{D}$ cardiac differentiation, respectively, until day 20 of differentiation. (B) Top GO terms for biological processes identified (FDR $<0.05$ ) for differentially upregulated genes $\left(\log _{2} \mathrm{FC}>1\right.$ and adjusted $p$-value $\left.<0.05\right)$ for $3 \mathrm{D}-\mathrm{D} 1$ versus hiPSC seeding, and $2 \mathrm{D}-\mathrm{D} 1$ versus hiPSC seeding. (C,D) Heat map highlighting the differentially expressed genes $\left(\log _{2} \mathrm{FC}>1\right.$ and adjusted p-value $<0.05$ ) related with the most significant GO terms for $(\mathbf{C}) 3 \mathrm{D}-\mathrm{D} 5$ vs 2D-D5 and (D) 3D-D9 vs 2D-D9. See Table S3 for full DE gene list. (E) Schematic representation highlighting the moment of begging of contraction in both 3D aggregates and 2D monolayer. (F) Expression profile of cardiac progenitor genes ISL1 and NKX2-5 throughout the entire process of cardiac differentiation. Values are normalized to GAPDH 
and relative to day 0 . Data are represented as mean $\pm S E M, n=3$ independent experiments. (G) Heat map highlighting the differentially expressed genes $\left(\log _{2} \mathrm{FC}<-1\right.$ and $\left.\log _{2} \mathrm{FC}>1\right)$ and adjusted $\mathrm{p}$-value $<0.05$ ) related with the most significant GO terms for 3D-D20 vs 2D-D20 differential expression analysis. See Table S3 for full DE gene list. (H) Representative section of 3D aggregates on day 20 of differentiation, highlighting the presence of CD $31^{+}$cells. Scale bars, $20 \mu \mathrm{m}$. (I) Replated CMs from dissociated 3D aggregates and 2D cardiac tissue at day 20 of differentiation, highlighting the prevalence of $\mathrm{SMA}^{+}$cells in the $2 \mathrm{D}$ culture when compared to $3 \mathrm{D}$ conditions. Scale bars, $50 \mu \mathrm{m}$ and $20 \mu \mathrm{m}$ in $2 \mathrm{D}$ and $3 \mathrm{D}$ conditions, respectively. See also Fig. S3.

9 of differentiation, the differential expression analysis between 3D and 2D cardiac differentiation conditions (Table S3 "DE gene list"), revealed an enrichment in 3D aggregates of GO terms related with cardiac conduction system and nodal/atrial muscle communication. Specifically, the protein-coding genes CACNA1C, GJA5, CACNA1G, SCN3B, KCNA5 and ANK2 were upregulated (Fig. 3D). In fact, most of these genes are described to be present in atrial CMs or in the nodal/conduction system myocytes. GJA5 gene, that encodes for the Cx40 gap junction, and KCNA5, that encodes for the ion channel Kv1.5, are described as cell markers for atrial CMs ${ }^{34,35}$, being almost absent in the ventricular working myocardium. Additionally, GJA5 is largely expressed in the conduction system myocytes, which includes the HIS bundles, the left and right bundle branches (LBB and RBB) and purkinje fibers, enabling a fast conduction of the impulse between the atrioventricular node (ANV) and ventricular working myocytes ${ }^{36}$. The calcium channel Cav3.1, encoded by CACNA1G, is preferentially expressed in nodal/ pacemaker myocytes although it is also abundantly expressed in atrial CMs and purkinje fibers ${ }^{37,38}$. Interestingly, it is at day $8 / 9$ of differentiation that $3 \mathrm{D}$ aggregates start to contract, in contrast with $2 \mathrm{D}$ monolayer culture, in which the beginning of contraction only starts at day 11/12 of differentiation (Fig. 3E). Despite this apparent earlier expression of structural and functional CM genes in 3D aggregates, RNA-seq data did not show a delay in the expression of cardiac progenitor markers, namely ISL1 and NKX2-5, in 2D monolayer vs 3D aggregates, a result further confirmed by qRT-PCR analysis (Fig. 3F).

At day 15 of differentiation, from a GO analysis of genes that were only upregulated in $3 \mathrm{D}$ aggregates or $2 \mathrm{D}$ monolayer, it was possible to observe that, in the case of 3D aggregates, regulation of heart contraction and cardiovascular system development appear as upregulated biological process, whereas in the case of 2D monolayer those terms were not represented and instead terms related with extracellular matrix organization and structure were highlighted (Fig. S3C).

A differential expression analysis comparing both cardiac tissues at day 20 of differentiation (Table S3 "DE gene list", "GO - 3D-D20 vs 2D-D20 - up") revealed an upregulation of genes related with endothelial differentiation/endothelium formation, such as KDR, CDH5, PECAM1, CD34 and GJA4 (Fig. 3G) in the 3D aggregates. The presence of $\mathrm{CD}_{3} 1^{+}$endothelial cells in $3 \mathrm{D}$ aggregates was further validated by immunostaining of aggregate sections (Fig. $3 \mathrm{H}$ ). In addition, genes involved in heart contraction, action potential and signal conduction, such as KCNJ11, GJA5, CACNA2D2, CACNA1G, ATP1A2, MYOM1 and MB, were also upregulated in the 3D aggregates, suggesting a higher degree of functional maturation. In the $2 \mathrm{D}$ monolayer cardiac tissue, different protein-coding genes of ECM were upregulated, including different collagens (COL1A1, COL4A1, COL5A1, COL9A1) lumican (LUM), laminin (LAMA5), fibronectin (FN1) and vitronectin (VTN). The upregulation of ECM protein-coding genes throughout the process of cardiac differentiation was already reported for 2D monolayer cardiac differentiation $^{39}$, as well as the upregulation in 2D monolayer compared with $3 \mathrm{D}$ aggregates ${ }^{17}$. The increased expression of these genes can be related with a higher content of fibroblast-like cells in the $2 \mathrm{D}$ monolayer since they are the main producers of $\mathrm{ECM}^{40}$. In addition, in $2 \mathrm{D}$ cardiac tissue, expression profiling results suggested the upregulation of protein-coding genes for skeletal muscle myocytes, namely TNNT1, and the upregulation of ACTN1 and TAGLN2, which suggests higher content of smooth muscle cells compared with the 3D cardiac aggregates. In fact, immunostaining of replated 3D aggregates and 2D monolayer suggests a higher prevalence of smooth muscle cells in the $2 \mathrm{D}$ culture system (Fig. 3I), which was further confirmed by $\mathrm{CALP}^{+}$cell quantification (Fig. 4D).

Performing the cardiac differentiation in 3D conditions impacts the cellular composition of cardiac aggregates and the maturity of cardiomyocytes. Taking into consideration the results obtained from the RNA-seq data regarding the transcriptional effect of 3D culture of hiPSCs prior to cardiac differentiation, we tested the effect of increasing the pre-differentiation period from 2 to 3 days, while maintaining the optimized D0 aggregate size (Fig. 4A). This increment resulted in a significant increase of CM differentiation efficiency from $\pm 70 \%$ to $>90 \%$ cTNT $^{+}(92 \pm 1 \%)$ cells after 15 days of differentiation (Fig. $\left.4 \mathrm{~B}\right)$, without compromising the expression of pluripotency markers OCT4, SSEA4 and TRA-1-60, at D0 (Fig. 4C). Also, increasing the pre-differentiation period improved the reproducibility among biological runs, which is still a common bottleneck in different reported studies of $2 \mathrm{D}$ cardiac differentiation, mainly the ones based on Wnt signalling modulation $^{41}$.

The 3D cardiac spheroids obtained with this improved 3D cardiac differentiation platform were then further characterized in terms of cell composition and compared to 2D monolayer. In 3D aggregates, apart from CMs, a small population of non-myocyte cells were detected at D15, comprising mainly CD $0^{+}$stromal cells $(3.3 \pm 0.1 \%$ $\mathrm{CD} 90^{+}$cells) (Fig. 4D). Additionally, immunostainning of different sections of cardiac aggregates shows the presence of different areas staining positively for the endothelial marker CD31 (Fig. 4E), demonstrating the existence of endothelial cells inside the aggregates. At day 30, aggregates show a decreased percentage of cTNT ${ }^{+}$cells, which can be attributed to the increased number of proliferative cells, particularly CD $90^{+}$fibroblast-like cells, which percentage increased to $\pm 6 \%$ at D30 (Fig. 4D). However, it can also be attributed to the observed decrease in total cell number ( $\pm 20 \%$ of cell loss) from D15 to D30 of differentiation, which potentially results from aggregates agglomeration. To avoid this phenomenon, two different modifications to the initial method were evaluated 
A

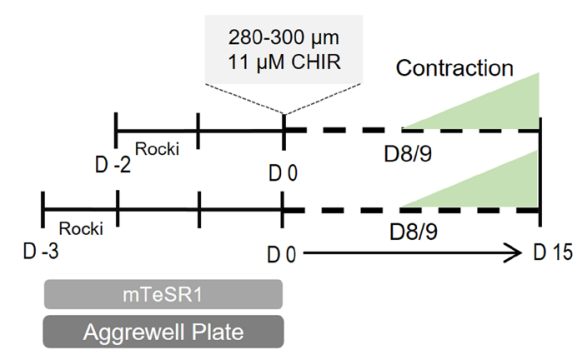

D

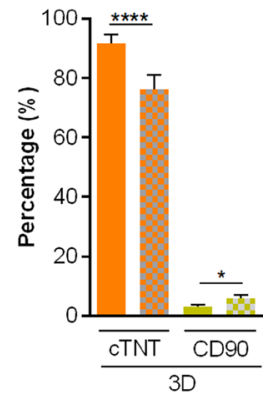

$\mathbf{F}$

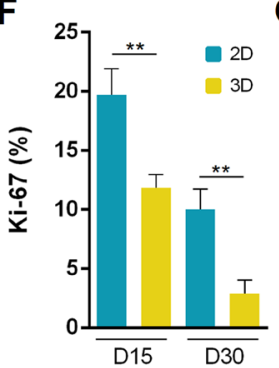

B
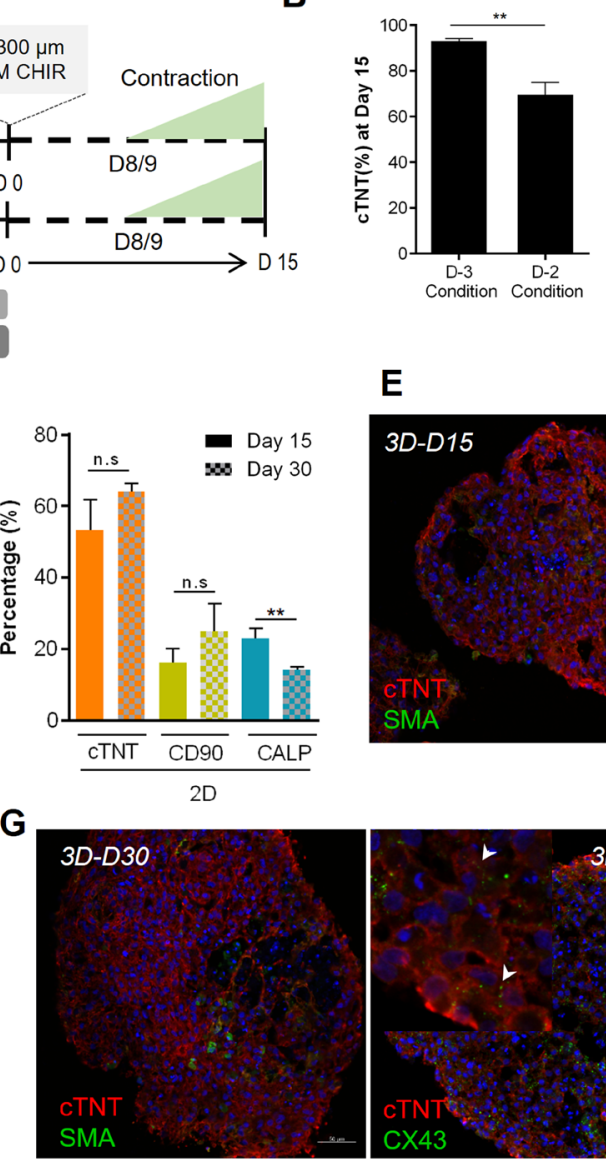

E

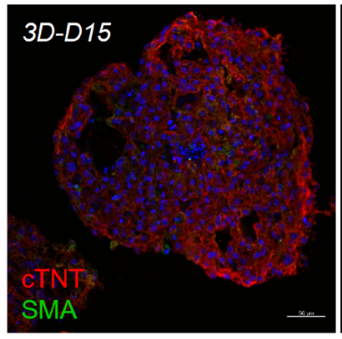

C

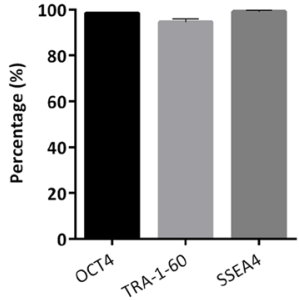

$3 D-D 15$
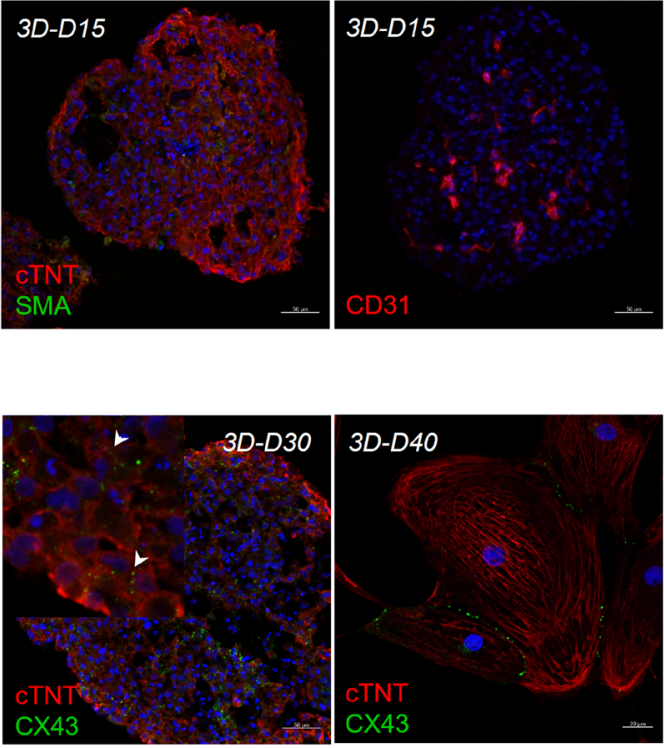

H

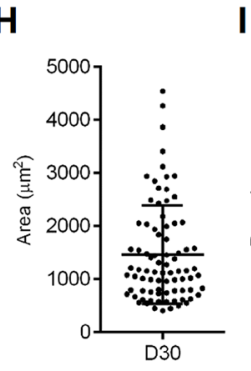

1

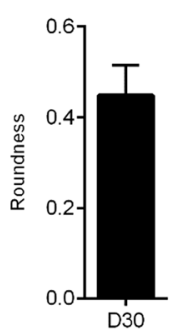

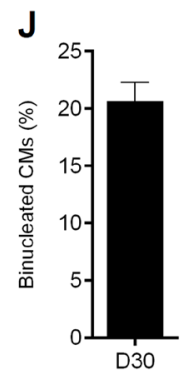

K

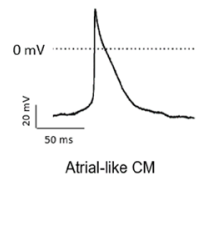

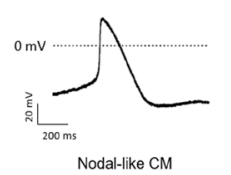

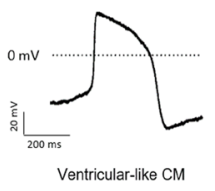

Figure 4. Performing the cardiac differentiation in 3D conditions impacts the cellular composition of cardiac aggregates and the maturity of cardiomyocytes. (A) Schematic representation highlighting the optimized 3D cardiac differentiation platform, in which hiPSC aggregates remain for 3 days in pluripotency maintenance medium before starting the differentiation process. The aggregate diameter at D0 was the same obtained with the previous version of the $3 \mathrm{D}$ platform (pre-differentiation period of 2 days) since the initial cell seeding density was decreased. (B) Cardiomyocyte differentiation efficiency in terms of cTNT ${ }^{+}$cells after 15 days of differentiation in both conditions, pre-differentiation period of 2 days (D-2) or 3 days (D-3). Data are represented as mean $\pm S E M$, $\mathrm{n}=6$ independent experiments. (C) Flow cytometry analysis of hiPSC 3D aggregates at D0 of differentiation, after 3 days in pluripotency maintenance media, for pluripotent transcription factor OCT4, and surface markers TRA-1-60 and SSEA4. Data are represented as mean \pm SEM, $\mathrm{n}=3$ independent experiments. (D) Quantification of different cell types present in 2D monolayer and 3D aggregates at D15 and D30 of differentiation (cTNT ${ }^{+}$ - CMs; CD90+ - Fibroblast-like cells; $\mathrm{CALP}^{+}$- Smooth Muscle cells). Data are represented as mean \pm SEM, at least $\mathrm{n}=3$ independent experiments. (E) Sections of 3D aggregates at day 15 of differentiation. Scale bars, $50 \mu \mathrm{m}$. (F) Percentage of CMs-Ki67 ${ }^{+}$cells in 3D aggregates and 2D monolayer at D15 and D30 of differentiation. Data are represented as mean \pm SEM, $\mathrm{n}=3$ independent experiments. (G) Sections of $3 \mathrm{D}$ aggregates at D30 of differentiation and D40 replated CMs. Scale bars, $50 \mu \mathrm{m}$, for sections, and $20 \mu \mathrm{m}$ in replated CMs. (H-J) Characterization of D30 replated CMs in terms of CMs area $(\mathbf{H})$, roundness $(\mathbf{I})$ (data are represented as mean, $\mathrm{n}=3$ ( 82 cells) independent experiments), and binucleation ( $\mathbf{J}$ ) (data are represented as mean, $\mathrm{n}=3$ ( 317 cells) independent experiments). (K) Representative traces of action potential (AP) recordings in day 30-35 of replated 3D aggregates of CMs, using whole cell patch clamp. $n=3$ independent experiments. See also Fig. S4. 
including (i) single-cell dissociation of the 3D aggregates at D10 of differentiation followed by re-aggregation in 96 well plates or (ii) direct transfer of the 3D aggregates to 96-well plates (Fig. S4A). Both approaches allowed the maintenance of viable 3D aggregates until D30 of cardiac differentiation (Fig. S4B,C). Comparing with 3D culture format, the efficiency of $2 \mathrm{D}$ cardiac differentiation was lower $\left(55 \pm 5 \% \mathrm{cTNT}^{+}\right.$cells $)$at D15 and a higher variability between biological runs was observed (Fig. 4D). In agreement with RNA-seq data, a higher prevalence of fibroblast-like cells ( $16 \pm 2 \% \mathrm{CD}^{+} 0^{+}$cells) and smooth muscle cells $\left(23 \pm 2 \% \mathrm{CALP}^{+}\right.$cells $)$was also confirmed in the $2 \mathrm{D}$ cardiac differentiation platform (Fig. 4D). Additionally, 2D cardiac monolayer differentiation lead to the detachment of contracting areas from the surface of culture matrix approximately after 20 days of culture, resulting in a considerable cell loss $( \pm 50 \%)$ and consequently reduced cell yield and higher variability. Additionally, using cTNT to specifically label cardiomyocytes and Ki67 as a marker of cell proliferation, we demonstrate the decrease in CMs-Ki67 ${ }^{+}$cells in both culture formats from D15 to D30. However, 3D aggregates show a significantly lower percentage of CMs staining positively for Ki67 at both analysed time points, decreasing from $11.9 \pm 0.6 \%$ at D15 to $2.9 \pm 0.7 \%$ at D30, whereas in $2 \mathrm{D}$ it decreases from $19.7 \pm 1.2 \%$ at D15 to $10.0 \pm 1 \%$ at D30 (Fig. 4F). This progressive exit from the cell cycle during maturation in 3D suggests a more mature phenotype compared to the one obtained in the 2D monolayer. Importantly, the expression of TNN13, which represents the more mature isoform of cardiac troponin $\mathrm{TNNI}^{42}$, is higher in 3D aggregates compared to 2D monolayer at D15 of differentiation (Figure S4D), reinforcing the idea of a higher degree of CM maturation in 3D cardiac aggregates at this time point.

After 4 weeks in culture, CMs from 3D cardiac aggregates show positive staining for connexin $43(\mathrm{Cx} 43)$ between neighbour CMs, which is an important gap junction present in the working myocardium (Fig. 4G). Additionally, CMs replated from D30 aggregates show an average area of $\pm 1,456 \mu \mathrm{m}^{2}$ and a roundness of \pm 0.45 , with $20.6 \pm 1.6 \%$ of binucleated CMs (Fig. $4 \mathrm{H}-\mathrm{J}$ ), which is in agreement with previous reports of CM in $3 \mathrm{D}$ culture $^{43,44}$. Finally, using the patch clamp technique, it was also possible to validate the electrophysiological activity of CMs dissociated from aggregates at day 30 of differentiation (Fig. $4 \mathrm{~K}$ ).

\section{Discussion}

When compared to $2 \mathrm{D}$ culture systems, 3D differentiation of hPSCs has proved to better mimic the process of embryogenesis in vivo, by recreating important spatial gradients of different signals that are essential for normal embryonic development, particularly for cardiogenesis ${ }^{14}$. Previous studies in the literature have mainly explored the implication of the 3D environment in cardiac tissue maturation, starting with a population of cardiac progenitor cells ${ }^{17}$ or CMs previously differentiated in $2 \mathrm{D}$ monolayer culture systems ${ }^{44-46}$. In a complementary approach to these studies, here we revealed important implications involved in transitioning the Wnt signalling-based 2D culture system to our novel integrated 3D culture platform, starting with forced and controlled aggregation of hiPSCs followed by their expansion, cardiac differentiation and maturation, in a 3D environment.

Oxygen gradients have been described as a fundamental physiological cue during organogenesis in the developing embryo ${ }^{47}$. In fact, due to oxygen diffusional limitations and also due to a non-established circulatory system, the development of the embryo in the early stages of embryogenesis occurs in a relatively oxygen-poor environment ${ }^{48}$. In our 3D culture platform, aggregates at day 0 of differentiation have already around $300 \mu \mathrm{m}$ of diameter, which can result in oxygen gradients since oxygen diffusion distance is estimated to be approximately $150 \mu \mathrm{m}^{21}$.

In this work we demonstrated that 3D culture of hiPSC before cardiac differentiation induced important regulatory changes that influenced the efficiency and robustness of cardiac differentiation. We attributed the 3D culture format itself and the consequent hypoxic response generated inside the aggregates as the major triggers for the transcriptional changes that we observed, namely (1) the stabilization of TGF- $\beta$ /Nodal pathway, (2) the upregulation of MAPK/JNK/ERK pathway and (3) the increase in glycolysis energy metabolism, which culminates in a balance between pluripotency maintenance and hiPSCs priming towards mesendoderm lineage differentiation. In fact, in the literature there are already evidences that suggest the priming of hPSCs towards mesoderm when cultured as $3 \mathrm{D}$ aggregates ${ }^{13,15}$. Also, HIF $1 \alpha$ has been described to indirectly induce NODAL transcription via Notch signal stabilization ${ }^{49,50}$, which can explain TGF- $\beta /$ Nodal pathway stabilization in our 3D hiPSC aggregates. The induction of mesoderm, during heart development, begins with high concentrations of NODAL in the proximal epiblast on mouse embryos ${ }^{51}$. Additionally, in vitro studies revealed that canonical Wnt and TGF- $\beta /$ Nodal signalling work together in the regulation of PS formation and suppression of NODAL prevents $\beta$-catenin mediated PS induction by CHIR addition ${ }^{52}$, reinforcing the importance of the Nodal signalling during the early stages of cardiac differentiation. Here we demonstrated that the initial stage of hiPSC culture as 3D hiPSC aggregates, before cardiac induction, influences the progression of differentiation, culminating in a faster and more efficient commitment of hPSCs towards cardiac mesoderm and consequently towards cardiomyocytes. We attribute this faster progression of cardiac differentiation in $3 \mathrm{D}$ aggregates, compared with $2 \mathrm{D}$ monolayer, not only to the differences in the initial cell population but also to the continuous hypoxic stimuli that may still be present throughout the differentiation process and the 3D structure itself, with increased cell-to-cell interaction.

Kinney and collegues ${ }^{53}$ referred in their work that forced aggregation allows a more homogeneous control of intercellular adhesion dynamics which may impact the differentiation capacity of ESCs. Particularly, they explored the relationship between E-cadherin, linked to cell-cell interaction, and $\beta$-catenin, involved in canonical Wnt signalling, which may be responsible for an enhanced cardiogenic differentiation capacity in 3D-EB-like aggregates. This fact can explain our RNA-seq data suggesting a higher degree of Wnt signaling activation in 3D aggregates at early stages of cardiac differentiation and also the upregulation of important targets of the Wnt/ $\beta$-catenin pathway, such as MESP1 and MEF2C. Additionally, previous studies suggest that hypoxia enhances the expression of mesodermal genes, acting as a mesoderm-inductive signal ${ }^{54}$, and also promote the activation of Wnt $/ \beta$-catenin signalling pathway ${ }^{55}$. Additionally, hypoxia, through HIF1 $\alpha$ stabilization, has been suggested to have, as direct targets, important cardiac transcriptional factors, such as $M E F 2 C^{56}$, which was upregulated in our 3D aggregates, 
and be also involved in the process of myofibrillogenesis ${ }^{56}$. In fact, different genes involved in cardiomyocyte structural and contraction apparatus, and also cardiac communication, including different gap junctions and ion channels genes, were upregulated at early stages of differentiation in 3D aggregates. This can potentially explain the earlier beginning of contraction in the 3D culture system compared with 2D monolayer.

Zhang and colleagues ${ }^{12}$, which also studied in parallel 2D and 3D cardiac differentiation systems, concluded that no major differences in the kinetics of cardiac differentiation became apparent between the 2D and 3D formats, suggesting a synchronized differentiation of the cells in both conditions. In that particular study, aggregates were induced at the same day that differentiation starts, contrary to what happens in our platform, where a pre-differentiation period is integrated with the differentiation platform and, as discussed before, has significant impact on the progression of differentiation. Also, Kerscher and colleagues ${ }^{57}$, which compared the differentiation into CMs from hPSCs encapsulated in a 3D hydrogel structure with a 2D monolayer culture system, reported that $\mathrm{CM}$ yield and gene expression level of cardiac markers were analogous to the ones observed in 2D monolayer for the same analysed time points. This reinforces the relevance and novelty of the integrated $3 \mathrm{D}$ cardiac differentiation platform that we developed.

Interestingly, some of the upregulated genes during the progression of differentiation and in the final 3D cardiac spheroids, compared with the $2 \mathrm{D}$ culture system, are involved in cardiac cell communication, and some of them are described to be preferentially expressed in atrial, nodal and conduction system CMs. This suggests that different and/or more functional CMs of different subtypes might be present in the 3D aggregates compared with the 2D monolayer. Since different subtypes of CMs are described to be originated from different subtypes of cardiac progenitor populations ${ }^{35}$, we suggest that the $3 \mathrm{D}$ environment is more prone to the development of a heterogeneous cardiac differentiation environment as a result of spatial gradients of molecules and oxygen inside the aggregates. In 2D monolayer culture systems cells are more homogenously exposed to the different stimuli, which can result in a more homogeneous differentiation towards a specific subtype of CMs. Taking into consideration the RNA-seq data, we cannot claim the existence of a different proportion or an enrichment in a specific CM subtype in each culture condition. However, a recently identified surface marker for ventricular-like cardiomyocyte progenitor cells, $L I F R^{58}$, was upregulated $(F C \pm 2.7)$ in the $2 \mathrm{D}$ culture at day 7 of differentiation compared to $3 \mathrm{D}$ aggregates. In fact, the $2 \mathrm{D}$ differentiation protocol based solely on the temporal modulation of Wnt signalling, has already been described to bias the cardiomyocyte differentiation towards ventricular-like cardiomyocytes ${ }^{59}$. A single-cell RNA-seq approach could enable the identification and characterization of the different cardiac progenitor populations that are potentially developed at the early stages of differentiation and of the different subtypes of cardiomyocytes present in the final 3D aggregates.

In conclusion, we developed a simple, highly efficient and robust 3D cardiac differentiation platform, using only the temporal modulation of Wnt signalling. This 3D integrated hiPSC expansion and differentiation platform contributes to a faster cardiac commitment of hiPSCs and to an earlier CM structural and functional maturation when compared with CMs obtained from monolayer culture. 2D differentiation of hiPSCs into cardiomyocytes using the Wnt signalling modulation, despite being a simple protocol, is a process that has a very high intrinsic variability ${ }^{41}$ ending up to be a poorly reliable process when the aim is to obtain CMs in a consistent manner for further applications. CMs produced with our 3D platform can be easily used for the development of in vitro engineered cardiac tissue (EHT) models, which in the majority of the reported cases uses hPSC-CMs previously differentiated in 2D culture platforms. With our 3D differentiation platform, hiPSC-CMs can be obtained in a faster, more efficient and reproducible way, and additionally CMs show already a higher degree of maturation compared to age-matched CMs obtained in 2D culture. The 3D platform allows the achievement of 20-25 million CMs at D10-D15 of cardiac differentiation per AggreWell ${ }^{\mathrm{TM}} 800$ plate, and overall this number can be easily increased using a scale out process.

\section{Experimental Procedures}

Cell maintenance. Human iPSCs were maintained in $\mathrm{mTeSR}^{\mathrm{TM}} 1$ medium (StemCell Technologies) on Matrigel-coated (Corning), tissue culture plates. Medium was changed daily. Cells were routinely passaged every three to four days using 0.5 mM EDTA solution (Thermo Fisher Scientific).

Cardiomyocyte differentiation in 2D and 3D culture conditions. For 2D monolayer culture, hiPSCs were seeded onto Matrigel-coated 12-well tissue culture plates and cultured in mTeSR $^{\mathrm{TM}} 1$. Culture medium was changed daily until a confluence of around $90-95 \%$ was attained. For 3D aggregates formation, hiPSCs were seeded on microwell plates (AggreWell ${ }^{\mathrm{TM}} 800$, StemCell Technologies) according to the manufacturer's instructions. For hiPSCs differentiation into cardiomyocytes, in both 2D and 3D culture conditions, an adapted GiWi protocol was used ${ }^{5}$. RPMI 1640 medium (Thermo Fisher Scientific) was used as basal medium. From day 0 to day 6, cells were cultured in RPMI medium supplemented with $2 \%(\mathrm{v} / \mathrm{v})$ B-27 minus insulin (Thermo Fisher Scientific), and from day 7 until the end of differentiation, cells were cultured in RPMI supplemented with $2 \%(v / v)$ B-27 (Thermo Fisher Scientific). At day 0 of differentiation, the Wnt signalling pathway was activated using the GSK3 inhibitor CHIR99021 (Stemgent) at a final concentration of $6 \mu \mathrm{M}$, in 2D conditions, and 3-18 $\mu \mathrm{M}$, in $3 \mathrm{D}$ conditions. After 24 hours, medium was changed to RPMI + B27 minus insulin. At day 3, cells were cultured in basal medium supplemented with Wnt inhibitor IWP-4 (Stemgent) at a final concentration of $5 \mu \mathrm{M}$, for two days. At day 7, medium was changed and in the case of 3D culture, aggregates were transferred to Ultra-Low Attachment plates (Corning). Thereafter, medium was changed every 3 days until cell harvest.

RNA sequencing and Data analysis. Total RNA from 2D monolayer and 3D aggregates at different stages of cardiac differentiation was extracted using High Pure RNA Isolation Kit (Roche), according to manufacturer's instructions. RNA libraries were prepared for sequencing using Lexogen QuantSeq $3^{\prime} \mathrm{mRNA}$-Seq Library Prep 
Kit FWD for Illumina using standard protocols. Sequencing was performed using Illumina HiSeq (50 cycle's protocol) or NextSeq (75 cycle's protocol) platforms. Sample read quality, reads mapping and counting were performed by a standard protocol from BlueBee Genomics Platform (http://www.bluebee.com/). With the RNA-Seq read counts matrix, we then used the DESeq2 (version 1.16.1) package of R to perform data normalization and differentially expressed genes (DEG) analysis. Information about DESeq2 package is available online at: https:// bioconductor.org/packages/release/bioc/html/DESeq2.html. Gene ontology (GO) terms were identified using the PANTHER (protein annotation through evolutionary relationship) classification system (version 13.1) (0. $^{6}$. GO terms were identified by analysing differentially expressed genes using the following settings: GO Biological Process, test type FISHER, reference list Homo Sapiens. Heat maps and PCA using a selection of enriched genes were generated in the web tool ClustVis ${ }^{61}$ and in $\mathrm{R}$.

Statistical analysis. Statistical significance was determined using a Student's t-test for all quantification except RNA-seq data. Data is represented as mean \pm SEM for at least three replicate samples (see figure legends for additional information). Differential gene expression analysis via DESeq. 2 for RNA-seq data is described in the section "RNA-seq Data Analysis".

Accession numbers. RNA-seq data for this study are available through Gene Expression Omnibus (GEO) Accession Number GSE116574.

\section{References}

1. Takeda, M. et al. Development of In Vitro Drug-Induced Cardiotoxicity Assay by Using Three-Dimensional Cardiac Tissues Derived from Human Induced Pluripotent Stem Cells. Tissue Eng. Part C Methods 24, 56-67 (2018).

2. Yang, X. \& Papoian, T. Moving beyond the comprehensive in vitro proarrhythmia assay: Use of human-induced pluripotent stem cell-derived cardiomyocytes to assess contractile effects associated with drug-induced structural cardiotoxicity. J. Appl. Toxicol. 1-11, https://doi.org/10.1002/jat.3611 (2018).

3. Birket, M. J. et al. Expansion and patterning of cardiovascular progenitors derived from human pluripotent stem cells. Nat. Biotechnol. 33, 970-979 (2015).

4. Kattman, S. J. et al. Stage-Specific Optimization of Activin/Nodal and BMP Signaling Promotes Cardiac Differentiation of Mouse and Human Pluripotent Stem Cell Lines. Cell Stem Cell 8, 228-240 (2011)

5. Lian, X. et al. Robust cardiomyocyte differentiation from human pluripotent stem cells via temporal modulation of canonical Wnt signaling. Proc. Natl. Acad. Sci. USA 109, E1848-57 (2012).

6. Rao, J. et al. Stepwise Clearance of Repressive Roadblocks Drives Cardiac Induction in Human ESCs. Cell Stem Cell 18, 341-53 (2016).

7. Ma, Z. et al. Self-organizing human cardiac microchambers mediated by geometric confinement. Nat. Commun. 6, 7413 (2015).

8. Warmflash, A., Sorre, B., Etoc, F., Siggia, E. D. \& Brivanlou, A. H. A method to recapitulate early embryonic spatial patterning in human embryonic stem cells. Nat. Methods 11, 847-854 (2014).

9. Kempf, H. et al. Controlling Expansion and Cardiomyogenic Differentiation of Human Pluripotent Stem Cells in Scalable Suspension Culture. Stem Cell Reports 3, 1132-1146 (2014).

10. Fonoudi, H. et al. A Universal and Robust Integrated Platform for the Scalable Production of Human Cardiomyocytes From Pluripotent Stem Cells. Stem Cells Transl. Med. 4, 1482-1494 (2015).

11. Chen, V. C. et al. Development of a scalable suspension culture for cardiac differentiation from human pluripotent stem cells. Stem Cell Res. 15, 365-375 (2015).

12. Zhang, M. et al. Universal cardiac induction of human pluripotent stem cells in two and three-dimensional formats: implications for in vitro maturation. Stem Cells 33, 1456-69 (2015).

13. Azarin, S. M. et al. Modulation of Wnt/ $\beta$-catenin signaling in human embryonic stem cells using a 3-D microwell array. Biomaterials 33, 2041-2049 (2012).

14. Mohr, J. C. et al. The microwell control of embryoid body size in order to regulate cardiac differentiation of human embryonic stem cells. Biomaterials 31, 1885-93 (2010).

15. Hsiao, C., Tomai, M., Glynn, J. \& Palecek, S. P. Effects of 3-D microwell culture on initial fate specification in human embryonic stem cells. AIChE J. 60, 1225-1235 (2014).

16. Husain, M. et al. Control of Human Embryonic Stem Cell Colony and Aggregate Size Heterogeneity Influences Differentiation Trajectories. Stem Cells 26, 2300-2310 (2008).

17. Correia, C. et al. 3D aggregate culture improves metabolic maturation of human pluripotent stem cell derived cardiomyocytes. Biotechnol. Bioeng. 115, 630-644 (2018).

18. Lian, X. et al. Robust cardiomyocyte differentiation from human pluripotent stem cells via temporal modulation of canonical Wnt signaling. Proc. Natl. Acad. Sci. 109, E1848-E1857 (2012).

19. Barbosa, H. S. C., Fernandes, T. G., Dias, T. P., Diogo, M. M. \& Cabral, J. M. S. New Insights into the Mechanisms of Embryonic Stem Cell Self-Renewal under Hypoxia: A Multifactorial Analysis Approach. PLoS One 7, e38963 (2012).

20. Dias, T. P. et al. Biophysical study of human induced Pluripotent Stem Cell-Derived cardiomyocyte structural maturation during long-term culture. Biochem. Biophys. Res. Commun. 499, 611-617 (2018).

21. Gatenby, R. A. \& Gillies, R. J. Why do cancers have high aerobic glycolysis? Nat. Rev. Cancer 4, 891-899 (2004)

22. Keith, B. \& Simon, M. C. Hypoxia-Inducible Factors, Stem Cells, and Cancer. Cell 129, 465-472 (2007).

23. Arthur, S. A., Blaydes, J. P. \& Houghton, F. D. Glycolysis Regulates Human Embryonic Stem Cell Self-Renewal under Hypoxia through HIF-2 $\alpha$ and the Glycolytic Sensors CTBPs. Stem cell reports 12, 1-15 (2019).

24. Varum, S. et al. Enhancement of human embryonic stem cell pluripotency through inhibition of the mitochondrial respiratory chain. Stem Cell Res. 3, 142-56 (2010).

25. Dhanasekaran, D. N. \& Reddy, E. P. JNK signaling in apoptosis. Oncogene 27, 6245-6251 (2008).

26. Moriyama, M. et al. BNIP3 upregulation via stimulation of ERK and JNK activity is required for the protection of keratinocytes from UVB-induced apoptosis. Cell Death Dis. 8, e2576 (2017).

27. Desireddi, J. R., Farrow, K. N., Marks, J. D., Waypa, G. B. \& Schumacker, P. T. Hypoxia Increases ROS Signaling and Cytosolic Ca $2+$ in Pulmonary Artery Smooth Muscle Cells of Mouse Lungs Slices. Antioxid. Redox Signal. 12, 595-602 (2010).

28. Chiquet, M. \& Flück, M. In Cell and Molecular Response to Stress. 2, 97-110 (2001).

29. Döring, Y., Pawig, L., Weber, C. \& Noels, H. The CXCL12/CXCR4 chemokine ligand/receptor axis in cardiovascular disease. Front. Physiol. 5, 212 (2014).

30. Kensah, G. et al. bFGF-mediated pluripotency maintenance in human induced pluripotent stem cells is associated with NRASMAPK signaling. Cell Commun. Signal. 16, 1-14 (2018).

31. Schier, A. F. Nodal morphogens. Cold Spring Harb. Perspect. Biol. 1, a003459 (2009). 
32. Pei, D., Xu, J., Zhuang, Q., Tse, H.-F. \& Esteban, M. A. Induced pluripotent stem cell technology in regenerative medicine and biology. Adv. Biochem. Eng. Biotechnol. 123, 127-41 (2010).

33. Ruttkay-Nedecky, B. et al. The role of metallothionein in oxidative stress. Int. J. Mol. Sci. 14, 6044-66 (2013).

34. Cyganek, L. et al. Deep phenotyping of human induced pluripotent stem cell-derived atrial and ventricular cardiomyocytes. JCI Insight 3, e99941 (2018).

35. Lee, J. H., Protze, S. I., Laksman, Z., Backx, P. H. \& Keller, G. M. Human Pluripotent Stem Cell-Derived Atrial and Ventricular Cardiomyocytes Develop from Distinct Mesoderm Populations. Cell Stem Cell 21, 179-194.e4 (2017).

36. van Weerd, J. H. \& Christoffels, V. M. The formation and function of the cardiac conduction system. Development 143, 197-210 (2016).

37. Liu, J., Laksman, Z. \& Backx, P. H. The electrophysiological development of cardiomyocytes. Adv. Drug Deliv. Rev. 96, 253-73 (2016).

38. Garg, P. et al. Human Induced Pluripotent Stem Cell-Derived Cardiomyocytes as Models for Cardiac Channelopathies. Circ. Res. 123, 224-243 (2018).

39. Wolling, H. et al. Quantitative Secretomics Reveals Extrinsic Signals Involved in Human Pluripotent Stem Cell Cardiomyogenesis. Proteomics 18, 1800102 (2018).

40. Fan, D., Takawale, A., Lee, J. \& Kassiri, Z. Cardiac fibroblasts, fibrosis and extracellular matrix remodeling in heart disease. Fibrogenesis Tissue Repair 5, 15 (2012)

41. Laco, F. et al. Unraveling the Inconsistencies of Cardiac Differentiation Efficiency Induced by the GSK3 $\beta$ Inhibitor CHIR99021 in Human Pluripotent Stem Cells. Stem cell reports 10, 1851-1866 (2018).

42. Bedada, F. B. et al. Acquisition of a quantitative, stoichiometrically conserved ratiometric marker of maturation status in stem cellderived cardiac myocytes. Stem Cell Reports 3, 594-605 (2014).

43. Correia, C. et al. Distinct carbon sources affect structural and functional maturation of cardiomyocytes derived from human pluripotent stem cells. Sci. Rep. 7, 8590 (2017).

44. Ronaldson-Bouchard, K. et al. Advanced maturation of human cardiac tissue grown from pluripotent stem cells. Nature 556, 239-243 (2018).

45. Mills, R. J. et al. Functional screening in human cardiac organoids reveals a metabolic mechanism for cardiomyocyte cell cycle arrest. Proc. Natl. Acad. Sci. USA 114, E8372-E8381 (2017).

46. Shadrin, I. Y. et al. Cardiopatch platform enables maturation and scale-up of human pluripotent stem cell-derived engineered heart tissues. Nat. Commun. 8 (2017).

47. Al-Mehdi, A.-B. et al. Perinuclear Mitochondrial Clustering Creates an Oxidant-Rich Nuclear Domain Required for HypoxiaInduced. Transcription. Sci. Signal. 5, ra47-ra47 (2012).

48. Mohyeldin, A., Garzón-Muvdi, T. \& Quiñones-Hinojosa, A. Oxygen in stem cell biology: a critical component of the stem cell niche. Cell Stem Cell 7, 150-61 (2010).

49. Quail, D. F. et al. Low oxygen levels induce the expression of the embryonic morphogen Nodal. Mol. Biol. Cell 22, 4809-21 (2011).

50. Gustafsson, M. V. et al. Hypoxia Requires Notch Signaling to Maintain the Undifferentiated Cell State. Dev. Cell 9, 617-628 (2005).

51. Burridge, P. W., Keller, G., Gold, J. D. \& Wu, J. C. Production of De Novo Cardiomyocytes: Human Pluripotent Stem Cell Differentiation and Direct Reprogramming. Cell Stem Cell 10, 16-28 (2012).

52. Funa, N. S. et al. $\beta$-Catenin Regulates Primitive Streak Induction through Collaborative Interactions with SMAD2/SMAD3 and OCT4. Cell Stem Cell 16, 639-652 (2015).

53. Kinney, M. A., Sargent, C. Y. \& McDevitt, T. C. Temporal Modulation of $\beta$-Catenin Signaling by Multicellular Aggregation Kinetics Impacts Embryonic Stem Cell Cardiomyogenesis. Stem Cells Dev. 22, 2665-2677 (2013).

54. Niebruegge, S. et al. Generation of human embryonic stem cell-derived mesoderm and cardiac cells using size-specified aggregates in an oxygen-controlled bioreactor. Biotechnol. Bioeng. 102, 493-507 (2009).

55. Mazumdar, J. et al. $\mathrm{O}_{2}$ regulates stem cells through Wnt/ $\beta$-catenin signalling. Nat. Cell Biol. 12, 1007-13 (2010).

56. Krishnan, J. et al. Essential role of developmentally activated hypoxia-inducible factor lalpha for cardiac morphogenesis and function. Circ. Res. 103, 1139-46 (2008).

57. Kerscher, P. et al. Direct hydrogel encapsulation of pluripotent stem cells enables ontomimetic differentiation and growth of engineered human heart tissues. Biomaterials 83, 383-95 (2016).

58. Foo, K. S. et al. Human ISL1 + Ventricular Progenitors Self-Assemble into an In Vivo Functional Heart Patch and Preserve Cardiac Function Post Infarction. Mol. Ther. 26, 1644-1659 (2018).

59. Lian, X. et al. Robust cardiomyocyte differentiation from human pluripotent stem cells via temporal modulation of canonical Wnt signaling. Proc. Natl. Acad. Sci. USA 109, E1848-57 (2012).

60. Mi, H., Muruganujan, A., Casagrande, J. T. \& Thomas, P. D. Large-scale gene function analysis with the panther classification system. Nat. Protoc. 8, 1551-1566 (2013).

61. Metsalu, T. \& Vilo, J. ClustVis: A web tool for visualizing clustering of multivariate data using Principal Component Analysis and heatmap. Nucleic Acids Res. 43, W566-W570 (2015).

\section{Acknowledgements}

RNA-sequencing was performed at Gene Expression Unit of Instituto Gulbenkian de Ciência, Oeiras, Portugal and CRG Genomics Unit, Barcelona, Spain. M.A.B. was supported by Fundação para a Ciência e Tecnologia (FCT) Portugal (PD/BC128362/2017). J.P.C. was supported by FCT Portugal (PD/BD135500/2018). The authors acknowledge financial support from FCT Portugal through iBB, Institute for Bioengineering and Biosciences, (UID/BIO/04565/2013) and from Programa Operacional Regional de Lisboa 2020 (Project No. 007317), PTDC/ EMD-TLM/29728/2017 and PTDC/EQU-EQU/29653/2017 projects, and project PRECISE - Accelerating progress toward the new era of precision medicine (PAC-PRECISE-LISBOA-01-0145-FEDER-016394, SAICTPAC/0021/2015). The authors also acknowledge funding from the European Union Framework Programme for Research and Innovation HORIZON 2020, under the TEAMING Grant agreement No. 739572 (H2020-WIDESPREAD-01-2016-2017).

\section{Author Contributions}

M.A.B., J.P.C., C.A.V.R., T.G.F., M.M.D. designed the study; M.A.B. and J.P.C. performed the differentiations; S.H.V. performed the calcium transient and electrophysiology analysis, L.M.M. was involved in RNA-seq data acquisition and M.A.B. in RNA-seq data analysis, M.A.B., S.H.V., L.M.M., J.M.S.C. and M.M.D. discussed the results and wrote the paper.

Additional Information

Supplementary information accompanies this paper at https://doi.org/10.1038/s41598-019-45047-9.

Competing Interests: The authors declare no competing interests. 
Publisher's note: Springer Nature remains neutral with regard to jurisdictional claims in published maps and institutional affiliations.

(c) (i) Open Access This article is licensed under a Creative Commons Attribution 4.0 International

License, which permits use, sharing, adaptation, distribution and reproduction in any medium or format, as long as you give appropriate credit to the original author(s) and the source, provide a link to the Creative Commons license, and indicate if changes were made. The images or other third party material in this article are included in the article's Creative Commons license, unless indicated otherwise in a credit line to the material. If material is not included in the article's Creative Commons license and your intended use is not permitted by statutory regulation or exceeds the permitted use, you will need to obtain permission directly from the copyright holder. To view a copy of this license, visit http://creativecommons.org/licenses/by/4.0/.

(c) The Author(s) 2019 